\title{
Exploring the numerical mind by eye-tracking: a special issue
}

\author{
Matthias Hartmann ${ }^{1} \cdot$ Martin H. Fischer ${ }^{2}$
}

Received: 7 February 2016/ Accepted: 11 February 2016/Published online: 29 February 2016

(C) Springer-Verlag Berlin Heidelberg 2016

\section{Introduction}

Psychological research has a long track record of developing tools for our understanding of mental processes. Prominent among these tools are the measurement of the duration of cognitive processes (mental chronometry) and the localization of these processes in the brain (cognitive neuroscience). Somewhat less prominent, but undeservedly so, is eye movement recording or eye-tracking. Eyetracking has a century-old history (Wade \& Tatler, 2005) that has recently culminated in the widespread availability of relatively affordable and low-effort tools for the unobtrusive study of visual exploratory behaviour (e.g., Holmqvist et al., 2011).

Eye movements, an endless succession of rapid jumps (saccades) and brief resting periods (fixations) of the eye balls, arguably constitute our most frequent goal-directed behaviour (cf. Desmurget, Pélisson, Rossetti, \& Prablanc, 1998; Hayhoe \& Ballard, 2005). Measurements of eye position can be taken several hundred times per second without requiring additional task instructions or interrupting the naturally occurring behaviour of participants. Successive measures with similar spatial coordinates are aggregated into one fixation with a specific location and duration; larger changes in location yield new fixations,

Matthias Hartmann

matthias.hartmann@psy.unibe.ch

$\triangle$ Martin H. Fischer

martinf@uni-potsdam.de

1 Department of Psychology, University of Bern, Fabrikstrasse 8, 3000 Bern, Switzerland

2 Division of Cognitive Sciences, University of Potsdam, Potsdam, Germany and thus information about saccade directions and saccade sizes. Multiple fixations falling on a given visual object or area of interest can be further aggregated, resulting in gaze durations or total viewing times or dwell times as additional eye movement measures (Holmqvist et al., 2011).

Importantly, just two simple assumptions about eye movement measures have allowed cognitive scientists to employ eye-tracking very productively in the service of cognitive research. First, gazing at something likely indicates the object of our thoughts (Tanenhaus, Spivey-Knowlton, Eberhard, \& Sedivy, 1995). And secondly, the time spent looking at an object corresponds to the time we think about this object (Just \& Carpenter, 1980). Both assumptions have received extensive support from reading research where most words are successively fixated and fixation durations primarily reflect ease of comprehension (e.g., Kliegl, Nuthmann, \& Engbert, 2006; Rayner, 1998; Rayner \& Reingold, 2015; Reichle, Pollatsek, Fisher, \& Rayner, 1998; Starr \& Rayner, 2001). Building on these simple assumptions, eye-tracking thus provides detailed information about the spatial selectivity and temporal extent of ongoing cognition, enabling researchers to "read the mind".

This research rationale can profitably be extended into the domain of numerical cognition. In this field of study the currently dominant view holds that number concepts are represented on a spatially oriented "mental number line", with small numbers represented to the left of larger numbers (at least in Western cultures; Dehaene, Bossini, \& Giraux, 1993). The "mental number line" gives rise to spatial-numerical associations, which can be widely observed, both in simple number classification tasks and also in more complex tasks such as mental arithmetic (Fischer \& Shaki, 2014). Eye movements provide online access to the focus of spatial attention and can therefore reveal the order and duration of concept activations, 
information that is not available when merely collecting response times or error rates at task completion. Moreover, eye movements can directly reflect strategy choices during task execution, thereby enabling a valid description of how a person solves a particular numerical problem; this will in turn help to understand why some persons may have more difficulties than others with mental arithmetic.

Despite this potential, relatively few eye-tracking studies in the domain of numerical cognition have so far been published when compared with the number of eye-tracking studies of language processing (we found approximately 50 vs. more than 1000 papers during the last 10 years, respectively). ${ }^{1}$ Researchers have only recently begun to make wider use of the advantages of eye-tracking in numerical cognition. This is reflected in the disproportionate increase in the number of published studies in the last few years (cf. Mock, Huber, Klein, \& Moeller, 2016, this issue) and also by an increased prevalence of eyetracking approaches to number processing on international conferences. An example of the latter trend was the symposium on "Numbers in the eye of the beholder: What eye movements reveal about numerical cognition" at the International Conference on Spatial Cognition (ICSC) in Rome in September 2015 (cf. Hartmann, 2015). Several speakers at this symposium were internationally leading researchers, many of whom contribute some of their best work to the present Special Issue.

The aim of this Special Issue is to update readers on current issues in numerical cognition and to highlight the added value of eye-tracking in this domain. The Special Issue brings together the work of nine teams of investigators from across the world. It opens with a review by Mock et al. (2016, this issue) of previous studies into systematic eye movements during number processing. The authors also introduce a processing model that considers the temporal dynamics of numerical cognition. Particularly, their model distinguishes an early stage of stimulus-driven processes from later, more top-down controlled stages of number processing. This model provides a helpful framework when interpreting past and future findings, independent of the methodology applied. The Special Issue further includes eye movement studies that focus on developmental and clinical aspects (Macci Cassia et al., 2016, this issue; van't Noordende, van Hoogmoed, Schot, \& Kroesbergen, 2016, this issue), as well as on spatial-numerical associations in single digit processing (Myachykov, Ellis,

\footnotetext{
1 These numbers are based on an online search of the Web of Knowledge database on Dec 20 2015, using the search term "eye movements" in conjunction with either "number" or "language". Research results that did not investigate aspects of numerical cognition or language processing were then manually removed to obtain the correct number of relevant studies between the years 2006 and 2015.
}

Cangelosi, \& Fischer, 2016, this issue; Ranzini, Lisi, \& Zorzi, 2016, this issue) and in mental arithmetic (Hartmann, Mast, \& Fischer, 2016, this issue; Yu et al., 2016, this issue). Further eye-tracking studies evaluate the influence of culture and language on numerical cognition (Bahnmueller et al., 2016, this issue), and individual differences within the healthy population (Ganor-Stern \& Weiss, 2016, this issue). We will briefly review the current state of research in these domains and highlight the novel contributions made with the help of eye-tracking.

\section{Development and impairments of number concepts}

The idea that humans and other animals are born with a "number sense" has attracted much attention during the last decades (Dehaene, 2011) and has in particular stimulated much research with infants. Eye movement analysis of the young mind is difficult but essential: when infants are not yet able to communicate through verbal language, analysing their spontaneous looking times at certain events can reveal underlying cognitive processes. For example, in the "violation of expectation"-paradigm, it is assumed that children look longer at stimuli that do not follow their expectation of how the world works. Wynn (1992) showed that infants look longer at a single doll that is revealed from behind an occluder when they previously observed an addition scenario (i.e., a second doll was added to an initial single doll, thus the expected solution would be "two") than when they previously observed a subtraction scenario (i.e., one doll was subtracted from a pair of dolls, thus the expected solution would be "one"). Moreover, the time infants (and also monkeys) spend looking at visual arrays follows Weber's Law (Cantlon \& Brannon, 2006; Libertus $\&$ Brannon, 2010). Furthermore, newborn infants preferentially look at arrays of visually presented objects that numerically match the number of auditory phonemes to which they have previously been familiarized (Izard, Sann, Spelke, \& Streri, 2009).

These results show that children are born with an inherent ability to discriminate between different magnitudes, and even with a basic understanding of arithmetic processes and abstract (in the sense of supra-modal) number concepts. Interestingly, there is also a preference for spatially ordered magnitudes early in childhood (de Hevia, Izard, Coubart, Spelke, \& Streri, 2014; De Hevia \& Spelke, 2010) and this preference is particularly pronounced for a left-to-right arrangement of magnitudes, both in children (Bulf, de Hevia, \& Macchi Cassia, 2015; de Hevia, Girelli, Addabbo, \& Cassia, 2014) and in newborn chicks (Rugani, Vallortigara, Priftis, \& Regolin, 2015; for a critical discussion see Shaki \& Fischer, 2015). In this issue, Macchi Cassia et al. (2016) continue this fascinating line of 
research by investigating the operational momentum $(\mathrm{OM})$ effect in infants. OM was originally described for nonsymbolic mental arithmetic in adults: when mentally adding or subtracting arrays of dots, participants are more willing to accept (incorrect) solutions with too many dots as correct for addition trials, and (incorrect) solutions with too few dots as correct for subtraction trials (McCrink, Dehaene, \& Dehaene-Lambertz, 2007). One possible explanation for this effect is that mental addition leads to an overshoot of a rightward movement along the mental number line, and subtraction to an overshoot of a leftward movement along the mental number line (McCrink et al., 2007), in analogy to the representational momentum effect showing that the vanishing position of a moving object is perceived further along the movement's trajectory (Hubbard, 2014). Macchi Cassia et al. (2016, this issue) study this effect further and habituate 12-month-old infants to ascending or descending sequences of different shapes; then they present them ascending or descending test sequences of new shapes that exhibited larger or smaller physical sizes. Infants look longer at the size changes that violate the OM effect. Thus, spontaneous eye behaviour in preverbal infants can reveal the presence of a spatial representation of continuous quantities already during the first year of life.

Yet another increasingly popular method to study children's representation of numbers is the "number-line estimation" task (also "number-to-position" task). In this task, a given number needs to be mapped onto its correct spatial position on a horizontal line (usually flanked by 0 at its left end and 100 at its right end; (Siegler \& Opfer, 2003). This task previously revealed that children have a compressed scaling of numbers, with relatively large distances between small numbers and smaller distances between larger numbers (e.g., the difference between 0 and 10 is larger than that between 90 and 100). Interestingly, children's performance on the number-line estimation task is associated with their mathematical performance, suggesting that the appropriate (uncompressed) mapping of numbers onto space might reflect better conceptual mathematical understanding (Booth \& Siegler, 2006). The use of eye-tracking during this task previously revealed specific strategies that are related to different developmental stages of numerical concepts: young children start from the endpoint of the line and count upward (or downward) in whole units until they reach the target position. This strategy is reflected in a linear distribution of fixations along the line (from one end to the final position). Older children start to count from the midpoint when the target position is closer to the midpoint than to one of the endpoints of the line. An increased use of such a midpoint strategy is associated with greater arithmetic competence (Schneider et al., 2008). Finally, adults use a fully proportion-based strategy: fixations are distributed along proportional reference points (e.g., endpoint, midpoint, points between the endpoint and midpoint; Sullivan, Juhasz, Slattery, \& Barth, 2011).

Increased knowledge about the "normal" development of numerical concepts in children (e.g., the age-dependent use of different strategies in the number-line estimation task) enables researchers to diagnose children with mathematical disabilities (van Viersen, Slot, Kroesbergen, Van't Noordende, \& Leseman, 2013). Van't Noordende et al. (2016, this issue) make use of the added value of eyetracking in order to better understand why children with developmental dyscalculia (i.e., difficulties in learning or comprehending arithmetic) perform worse in the numberline estimation task than children with typical mathematical development. They find that dyscalculic children attend to different features of the number line: they make less efficient use of reference points and are less capable of adapting their strategy when compared to age-matched controls. This study shows that eye-tracking reveals useful additional information about strategy use of children with mathematical impairments, which might be helpful for the development of interventions.

\section{Associations between numbers and space}

Dehaene et al. (1993) famously measured the speed of odd-even decisions (parity task) for single digits with lateralized button presses and discovered that responses to small numbers were faster on the left than on the right side, and vice versa for large numbers. This so-called SNARC (spatial-numerical association of response codes) effect has been taken as evidence that numbers are represented in an ordered fashion along a spatially oriented mental number line, with small numbers to the left of larger numbers. The discovery of a systematic association between the seemingly abstract concept of numbers and our physical space has since attracted much attention in the cognitive sciences. To illustrate, the seminal study by Dehaene et al. (1993) has been cited more than 1690 times (source: www. google.scholar.com, 6. Feb. 2016). SNARC-like effects have been investigated in a wide range of numerical tasks (Fischer \& Shaki, 2014), and can be observed across different effectors, including hands and feet (Schwarz \& Müller, 2006) and also the eyes (e.g., Schwarz \& Keus, 2004). For example, eye movements to the left side are faster after processing a small number, whereas eye movements to the right side are faster after processing a large number, when compared to the opposite pairings. Such "oculomotor SNARC effects" are not only reflected in saccadic response times, but also in spontaneously adopted gaze positions. Loetscher et al. (2010) showed that 
eye gaze position in the dark drifts rightward and upward when relatively larger numbers (i.e., numbers preceded by a smaller number) are called out in a random number generation task; similarly, the participant's gaze drifts leftward and downward for relatively small numbers). In this issue, Myachykov et al. (2016) show that such numberinduced ocular drifts occur even then when participants fixate a central visual stimulus on the screen (Experiment 1) or when they are engaged in an additional saccade task (Experiment 2), suggesting that the processing of numerical magnitudes leads to inescapable interference with eye movement control (a phenomenon they labelled oculomotor resonance effect). Further evidence for such interference between semantic and oculomotor mechanisms comes from Ranzini et al. (2016, this issue), showing that voluntary leftward and rightward eye movements (during smooth or stepwise pursuit of lateral targets) modulate the processing of number magnitudes in a parity judgment task. These results confirm previous claims of a bi-directional link between physical and representational numerical space (cf. Fischer, Castel, Dodd, \& Pratt, 2003; Hartmann, Grabherr, \& Mast, 2012; Zorzi, Priftis, \& Umilta, 2002).

The studies reported so far have assessed spatial-numerical associations for the processing of single numbers. However, spatial-numerical associations can also be found for more complex numerical tasks, such as mental arithmetic (Fischer \& Shaki, 2014). Therefore, it is not unexpected to see systematic eye movement patterns during such tasks. Hartmann et al. (2016, this issue) find that eye gaze position on a blank screen shifts upward and rightward when participants perform continuous additions (i.e., upward counting), supporting the idea that mental addition is conceptualized as rightward movement along the mental number line (McCrink et al., 2007; Pinhas \& Fischer, 2008). By presenting addition and subtraction problems and varying the operand order, Yu et al. (2016, this issue) find further support for this claim and clarify that the direction of the spatial-numerical association during mental arithmetic does not only depend on the operation (addition-right, subtraction-left), but also on the relative magnitude of operands and results (see also Hartmann, Mast, \& Fischer, 2015; Loetscher, Bockisch, \& Brugger, 2008). These results point to a surprisingly flexible mental number line in the context of arithmetic.

Noteworthy is furthermore the fact that some of the studies in this issue make use of the high temporal resolution of eye movement recording to describe the exact time course of the spatial-numerical associations (Hartmann et al., 2016; Myachykov et al., 2016; Yu et al., 2016). Interestingly, Hartmann et al. find a different time course for horizontal than for vertical spatial-numerical associations, suggesting that they might be governed by different mechanisms (for further discussions see Fischer, 2012;
Hartmann, Gashaj, Stahnke, \& Mast, 2014; Hartmann et al., 2015; Winter, Matlock, Shaki, \& Fischer, 2015). Myachykov et al. (2016, this issue) interpret their multiphasic oculomotor resonance effect to suggest that the spatial mapping might consist of distinct processing stages.

\section{Language shapes numerical cognition}

Culture, and in particular written language, plays an important role in shaping the spatial representation of ordered dimensions such as numbers (e.g., Göbel, 2015; Göbel, Shaki, \& Fischer, 2011). It has been shown already in the seminal paper by Dehaene et al. (1993) that adults from a right-to-left reading culture had a smaller or even reversed SNARC effect compared to adults from Western cultures. Shaki, Fischer, and Petrusic (2009) compared three cultures and found that reading habits for both numbers and words determine the direction and strength of the SNARC effect. But even for a given reading direction, the positioning of numbers within a text will quickly modulate their spatial associations (Fischer, Mills, \& Shaki, 2010).

Besides the influence on the SNARC effect, language also determines the order of naming the decade and unit of multi-digit numbers. For example, when reading the number "52", English speakers first mention the decade value, followed by the unit ("fifty-two"), whereas the opposite is true for German speakers ("zwei-und-fünfzig", "two-and-fifty”). Moeller, Shaki, Göbel, and Nuerk (2015) recently reported systematic influences of reading/writing direction as well as number word formation (non-inverted as in the English or Hebrew languages vs. inverted as in the German and Arabic languages) in a magnitude comparison task with two-digit numbers. Eye-tracking is a helpful tool to study such effects: it allows assessing whether one's attention goes first to the decade or the unit digit. Eye movement analysis confirmed that multi-digit numbers are not perceived as an entity but are decomposed and processed in parallel: more and earlier fixations are found on the decade and somewhat fewer fixations on the unit (Moeller, Fischer, Nuerk, \& Willmes, 2009). However, for the processing of numbers larger than 99 it has been argued that an additional sequential processing stage is involved because numbers become too wide to be processed entirely in parallel (Meyerhoff, Moeller, Debus, \& Nuerk, 2012). In their contribution to the present Special Issue, Bahnmueller et al. (2016) use eye-tracking to further study such language-related influences (for German vs. English) on the processing of three-digit numbers (e.g., 742_896). The observed eye gaze pattern generally confirms a parallel processing style for both languages. Interestingly, a subgroup of their German-speaking participants shows a 
reverse hundred-decade-compatibility effect, as is evident from longer inspection times for compatible than incompatible number pairs (in the example above the hundreddecade relationship is compatible because $7<8$ and $4<9)$. This result can be interpreted as reflecting sequential number processing (cf. Nuerk, Weger, \& Willmes, 2002) and therefore the findings of this study point to sequential processing in some German speakers but not in English speakers. This difference can be attributed to different language characteristics: The German language is generally associated with a more sequential reading style (letter-by-letter reading) when compared to the English language (Rau, Moll, Snowling, \& Landerl, 2015). Bahnmueller et al.'s (2016) findings therefore broaden the scope of language influences on number processing.

\section{Variation in numerical performance}

Eye-tracking reveals how a person solves a particular numeric problem. Some studies described above have used eye-tracking to better understand differences in problemsolving strategies between normally functioning individuals compared to those with mathematical difficulties (e.g., Van't Noordende et al., 2016, this issue). Such an approach might also be useful to study non-clinical populations, for example to extract and identify advantageous task strategies applied by particularly well-performing individuals, or to track the success of interventions within a given individual.

In a seminal oculomotor approach to problem solving, Grant and Spivey (2003) have shown that eye movements can prime certain solutions. Specifically, they asked participants to solve Duncker's (1935/1963) radiation problem, which requires destroying a tumour with a laser beam without injuring the surrounding healthy tissue. The solution requires firing multiple low-intensity lasers from different directions so that they converge on the tumour. For different groups of participants, the authors highlighted either the tumour or the tissue, thus inducing different eye movement patterns while looking at the problem sketch. Solution rates doubled when highlighting the tissue, presumably because the resulting eye movements primed different paths from the outside towards the tumour (see also Thomas \& Lleras, 2007).

Susac et al. (2014) investigated problem-solving strategies in the context of mental arithmetic. They assessed participants' eye movements when they rearranged algebraic equations, and participants also reported their strategies after the completion of the task. The authors found a correlation between the number of fixations and participants' efficiency in equation solving. Crucially, eye movement data were found to be more objective and reliable than the participants' explicit reports, showing that eye-tracking provides insights into otherwise unavailable task strategies.

The final study of this Special Issue by Ganor-Stern and Weiss (2016) further illustrates the merits of this approach. The authors report effects of practice on students' estimation performance for multi-digit multiplications. Participants judge whether the correct result would be smaller or larger than a given reference number. With increasing practice, dwell times and the frequency of first fixations on the reference number increase, reflecting a specific change in task strategy. This study nicely illustrates how eye movement analyses can help identify different problemsolving strategies in the domain of numerical cognition.

\section{Conclusions and outlook}

The studies reported here all highlight the added value of eye-tracking when exploring the numerical mind. Eye movement recording yields access to the infant's mind or provides information about specific task strategies that cannot be extracted from response times or error rates alone (Bahnmueller et al., 2016; Ganor-Stern and Weiss, 2016; Macci Cassia et al., 2016; Van't Noordende et al., 2016). Moreover, the studies reported here shed new light on the temporal development of spatial-numerical associations during single number processing and arithmetic performance (Hartmann et al., 2016; Myachykov et al., 2016; Yu et al., 2016). The findings of these latter studies point toward an even richer role of eye movement research within the cognitive sciences. The principal function of eye movements is to bring details of our visual environment into focus, allowing for conscious perception. However, our eyes do not only move to extract information from the visual world: previous studies already showed that inspecting a mental image in the mind's eye (e.g., inspecting an imagined house from the bottom to the top) leads to corresponding eye movements in the physical world (Laeng \& Teodorescu, 2002; Spivey \& Geng, 2001), and eye movement patterns during memory retrieval resemble those during real-time exploration (Johansson, Holsanova, Dewhurst, \& Holmqvist, 2012; Johansson \& Johansson, 2014; Martarelli \& Mast, 2013; Micic, Ehrlichman, \& Chen, 2010). The studies reported here suggest that the eyes also "inspect" abstract concepts such as a mental number line, and that they "act out" spatial relations of our thoughts. Such internally driven eye movements might thus reflect the fundamental fact that human cognition is "embodied", i.e. inevitably grounded in sensorimotor experiences in physical space (Coello \& Fischer, 2016; Fischer \& Coello, 2016). This fact is also expressed in the ubiquitous correlation between quantity 
and verticality that inspired universal linguistic expressions (e.g., "more is up" or "high price"; Lakoff \& Johnson, 1980). However, the exact role of such internally driven eye movements remains speculative at present. Particularly, it is not clear whether they are epiphenomenal to the recruitment of a mental number line or whether they play a functional role in the understanding of quantity. The finding that number processing is impaired when incongruent eye movements are performed (Ranzini et al., 2016) points to the latter possibility, although it still needs to be clarified whether the eye movements, or rather the lateral cues, are responsible for this interference effect (cf. Masson \& Pesenti, 2015). A further argument in favour of a functional role of eye movements is provided through the neuroanatomical links between cortical oculomotor control structures and magnitude networks. Numbers are processed within a generalized magnitude system in the parietal cortex (Bueti \& Walsh, 2009; Walsh, 2003, 2014), an area that is also involved in the processing of spatial information and planning of saccades (e.g., Cohen \& Andersen, 2002; Nyffeler, Hartmann, Hess, \& Müri, 2008). Moreover, Knops, Thirion, Hubbard, Michel, and Dehaene (2009) found partially overlapping brain activity when performing a lateral saccade task and a mental arithmetic task, namely for leftward saccades and subtraction, and for rightward saccades and addition. Thus, beyond a conceptual link between numbers and space, eye movement planning and number processing interact at the neuronal level (Burr, Ross, Binda, \& Morrone, 2010). Combined eye-tracking and neuroimaging techniques might therefore be helpful to further explore the shared network for numbers and eye movement control.

It is also important to point out that the full potential of eye-tracking in the study of numerical cognition has not yet been exhausted. For example, exploring individual differences in numerical performance by eye-tracking has mostly been limited to the clinical domain, but there is also much potential to study the "healthy" mind, for example to better describe how complex numerical tasks are solved, and how good performers differ in their strategy from less good performers. To this end, the application of new paradigms might be useful. For example, the adaptation of paradigms traditionally used to study language comprehension, such as the visual world paradigm (Huettig \& Altmann, 2005; Huettig, Rommers, \& Meyer, 2011), might be adapted for the numerical domain: investigating eye movements when several numbers are simultaneously presented on a screen might provide further insights into the temporal sequence of thoughts when solving numerical problems (Hartmann, Laubrock, \& Fischer, in preparation; Hintz \& Meyer, 2015). The knowledge of successful task strategies derived from eye movement analyses (cf. Ganor-Stern and Weiss, 2016; van't Noordende et al., 2016) might then be used for the development of training interventions; in turn, the success of these interventions can then be determined through eye-tracking.

There is also great potential in the use of additional eyetracking parameters. Besides fixations and saccades, most eye trackers provide information about the size of the pupil and the occurrence of so-called micro-saccades. The pupil size reflects cognitive load (Kahneman \& Beatty, 1966) and this eye-based measure of cognition was already utilized in early studies of mental arithmetic (Hess \& Polt, 1964). Pupillometry can also be used to measure shifts in covert spatial attention, task expectations, or mind wandering (for a recent review see Hartmann \& Fischer, 2014), which might all be important factors when studying numerical cognition. Another useful eye-tracking measurement are micro-saccades, small changes of eye position during an eye fixation that prevent visual information from decaying (Martinez-Conde, Macknik, Troncoso, \& Dyar, 2006). Micro-saccades have also been related to shifts in spatial attention (Engbert \& Kliegl, 2003), and more recently to task difficulty during mental arithmetic (Gao, Yan, \& Sun, 2015; Siegenthaler et al., 2014): micro-saccade rate decreases and their magnitude increases with increasing task difficulty. Thus, pupil size and micro-saccades might be helpful in explaining variance in performance resulting from task difficulty or from individual factors during each trial (e.g., focus of attention, task compliance).

This Special Issue highlights how the eyes can be used as a window onto the mathematical mind, and will hopefully further promote the use of eye-tracking in numerical cognition research.

Acknowledgments We thank the Editor-in-Chief, Bernhard Hommel, for supporting this Special Issue in Psychological Research. We thank all authors and reviewers for their valuable contribution to this issue. M.H. was funded by the Swiss National Science Foundation (P2BEP1_152104).

\section{References}

Bahnmueller, J., Huber, S., Nuerk, H. -C., Göbel, S. M., Moeller, K. (2016). Processing multi-digit numbers: A translingual eye tracking study. Psychological Research, (online first version).

Booth, J. L., \& Siegler, R. S. (2006). Developmental and individual differences in pure numerical estimation. Developmental Psychology, 42(1), 189-201. doi:10.1037/0012-1649.41.6.189.

Bueti, D., \& Walsh, V. (2009). The parietal cortex and the representation of time, space, number and other magnitudes. Philosophical Transactions of the Royal Society B: Biological Sciences, 364(1525), 1831-1840.

Bulf, H., de Hevia, M. D., \& Macchi Cassia, V. (2015). Small on the left, large on the right: Numbers orient visual attention onto space in preverbal infants. Developmental Science, . doi:10.1111/ desc.12315. (online first version). 
Burr, D. C., Ross, J., Binda, P., \& Morrone, M. C. (2010). Saccades compress space, time and number. Trends in Cognitive Science, 14(12), 528-533. doi:10.1016/j.tics.2010.09.005.

Cantlon, J. F., \& Brannon, E. M. (2006). Shared system for ordering small and large numbers in monkeys and humans. Psychological Science, 17(5), 401-406. doi:10.1111/j.1467-9280.2006.01719. $\mathrm{x}$.

Coello, Y., \& Fischer, M. H. (2016). Foundations of embodied cognition: Volume 1. Perceptual and emotional embodiment. London: Taylor \& Francis.

Cohen, Y. E., \& Andersen, R. A. (2002). A common reference frame for movement plans in the posterior parietal cortex. Nature Reviews Neuroscience, 3(7), 553-562.

de Hevia, M. D., Girelli, L., Addabbo, M., \& Cassia, V. M. (2014a). Human infants' preference for left-to-right oriented increasing numerical sequences. PLoS One, 9(5), e96412.

de Hevia, M. D., Izard, V., Coubart, A., Spelke, E. S., \& Streri, A. (2014b). Representations of space, time, and number in neonates. Proceedings of the National Academy of Sciences, 111(13), 4809-4813.

De Hevia, M. D., \& Spelke, E. S. (2010). Number-space mapping in human infants. Psychological Science, 21(5), 653-660.

Dehaene, S. (2011). The number sense: How the mind creates mathematics. Oxford: Oxford University Press.

Dehaene, S., Bossini, S., \& Giraux, P. (1993). The mental representation of parity and number magnitude. Journal of Experimental Psychology: General, 122(3), 371-396.

Desmurget, M., Pélisson, D., Rossetti, Y., \& Prablanc, C. (1998). From eye to hand: Planning goal-directed movements. Neuroscience and Biobehavioral Reviews, 22(6), 761-788.

Duncker, K. (1935/1963). Zur Psychologie des produktiven Denkens [The psychology of productive thinking]. Berlin, Germany: Springer.

Engbert, R., \& Kliegl, R. (2003). Microsaccades uncover the orientation of covert attention. Vision Research, 43(9), 1035-1045.

Fischer, M. H. (2012). A hierarchical view of grounded, embodied, and situated numerical cognition. Cognitive Processing, 13(1), 161-164.

Fischer, M. H., Castel, A. D., Dodd, M. D., \& Pratt, J. (2003). Perceiving numbers causes spatial shifts of attention. Nature Neuroscience, 6(6), 555-556. doi:10.1038/nn1066nn1066.

Fischer, M. H., \& Coello, Y. (2016). Foundations of embodied cognition: Volume 2. Conceptual and interactive embodiment. London: Taylor \& Francis.

Fischer, M. H., Mills, R. A., \& Shaki, S. (2010). How to cook a SNARC: Number placement in text rapidly changes spatialnumerical associations. Brain and Cognition, 72(3), 333-336. doi:10.1016/j.bandc.2009.10.010.

Fischer, M. H., \& Shaki, S. (2014). Spatial associations in numerical cognition-from single digits to arithmetic. Quarterly Journal of Experimental Psychology (Hove), 67(8), 1461-1483. doi:10. 1080/17470218.2014.927515.

Ganor-Stern, D., \& Weiss, N. (2016). Tracking practice effects in computation estimation. Psychological Research, (online first version).

Gao, X., Yan, H., \& Sun, H. (2015). Modulation of microsaccade rate by task difficulty revealed through between- and within-trial comparisons. Journal of Vision, 15(3), 1-15.

Göbel, S. M. (2015). Up or down? Reading direction influences vertical counting direction in the horizontal plane: A crosscultural comparison. Frontiers in Psychology, 6228.

Göbel, S. M., Shaki, S., \& Fischer, M. H. (2011). The cultural number line: A review of cultural and linguistic influences on the development of number processing. Journal of Cross-Cultural Psychology, 42(4), 543-565.
Grant, E. R., \& Spivey, M. J. (2003). Eye movements and problem solving guiding attention guides thought. Psychological Science, 14(5), 462-466.

Hartmann, M. (2015). Numbers in the eye of the beholder: What do eye movements reveal about numerical cognition? Cognitive Processing, 16(1), 245-248. doi:10.1007/s10339-015-0716-7.

Hartmann, M., \& Fischer, M. H. (2014). Pupillometry: The eyes shed fresh light on the mind. Current Biology, 24(7), R281-R282. doi:10.1016/j.cub.2014.02.028.

Hartmann, M., Gashaj, V., Stahnke, A., \& Mast, F. (2014). There is more than "more is up": Hand and foot responses reverse the vertical association of number magnitudes. Journal of Experimental Psychology: Human Perception and Performance, 40(4), 1401-1414. doi:10.1037/a0036686.

Hartmann, M., Grabherr, L., \& Mast, F. W. (2012). Moving along the mental number line: Interactions between whole-body motion and numerical cognition. Journal of Experimental Psychology: Human Perception and Performance, 38(6), 1416-1427. doi:10. 1037/a0026706

Hartmann, M., Laubrock, J., \& Fischer, M. H. (in preparation). The visual number world: A new approach to study the mathematical mind.

Hartmann, M., Mast, F. W., \& Fischer, M. H. (2016). Counting is a spatial process: Evidence from eye movements. Psychological Research. doi:10.1007/s00426-015-0722-5. (online first version).

Hartmann, M., Mast, F. W., \& Fischer, M. H. (2015). Spatial biases during mental arithmetic: Evidence from eye movements on a blank screen. Frontiers in Psychology, 6, 12. doi:10.3389/fpsyg. 2015.00012

Hayhoe, M., \& Ballard, D. (2005). Eye movements in natural behavior. Trends in Cognitive Science, 9(4), 188-194.

Hess, E. H., \& Polt, J. M. (1964). Pupil size in relation to mental activity during simple problem-solving. Science, 143(3611), 1190-1192.

Hintz, F., \& Meyer, A. S. (2015). Prediction and production of simple mathematical equations: Evidence from visual world eye-tracking. PLoS One, 10(7), e0130766.

Holmqvist, K., Nyström, M., Andersson, R., Dewhurst, R., Jarodzka, H., \& Van de Weijer, J. (2011). Eye tracking: A comprehensive guide to methods and measures. Oxford: Oxford University Press.

Hubbard, T. L. (2014). Forms of momentum across space: Representational, operational, and attentional. Psychonomic Bulletin \& Review, 21(6), 1371-1403.

Huettig, F., \& Altmann, G. T. (2005). Word meaning and the control of eye fixation: Semantic competitor effects and the visual world paradigm. Cognition, 96(1), B23-B32.

Huettig, F., Rommers, J., \& Meyer, A. S. (2011). Using the visual world paradigm to study language processing: A review and critical evaluation. Acta Psychologica (Amst), 137(2), 151-171.

Izard, V., Sann, C., Spelke, E. S., \& Streri, A. (2009). Newborn infants perceive abstract numbers. Proceedings of the National Academy of Sciences, 106(25), 10382-10385.

Johansson, R., Holsanova, J., Dewhurst, R., \& Holmqvist, K. (2012). Eye movements during scene recollection have a functional role, but they are not reinstatements of those produced during encoding. Journal of Experimental Psychology: Human Perception and Performance, 38(5), 1289.

Johansson, R., \& Johansson, M. (2014). Look here, eye movements play a functional role in memory retrieval. Psychological Science, 25(1), 236-242. doi:10.1177/0956797613498260.

Just, M. A., \& Carpenter, P. A. (1980). A theory of reading: From eye fixations to comprehension. Psychological Review, 87(4), 329.

Kahneman, D., \& Beatty, J. (1966). Pupil diameter and load on memory. Science, 154, 3. 
Kliegl, R., Nuthmann, A., \& Engbert, R. (2006). Tracking the mind during reading: The influence of past, present, and future words on fixation durations. Journal of Experimental Psychology: General, 135(1), 12.

Knops, A., Thirion, B., Hubbard, E. M., Michel, V., \& Dehaene, S. (2009). Recruitment of an area involved in eye movements during mental arithmetic. Science, 324(5934), 1583-1585. doi:10.1126/science.1171599.

Laeng, B., \& Teodorescu, D.-S. (2002). Eye scanpaths during visual imagery reenact those of perception of the same visual scene. Cognitive Science, 26(2), 207-231.

Lakoff, G., \& Johnson, M. (1980). Conceptual metaphor in everyday language. The Journal of Philosophy, 77(8), 453-486.

Libertus, M. E., \& Brannon, E. M. (2010). Stable individual differences in number discrimination in infancy. Developmental Science, 13(6), 900-906.

Loetscher, T., Bockisch, C. J., \& Brugger, P. (2008). Looking for the answer: The mind's eye in number space. Neuroscience, 151(3), 725-729. doi:10.1016/j.neuroscience.2007.07.068.

Loetscher, T., Bockisch, C. J., Nicholls, M. E., \& Brugger, P. (2010). Eye position predicts what number you have in mind. Current Biology, 20(6), R264-R265. doi:10.1016/j.cub.2010.01.015.

Macchi Cassia, V., McCrink, K., de Hevia, M. D., Gariboldi, V., \& Bulf, H. (2016). Operational momentum and size ordering in preverbal infants. Psychological Research, (online first version).

Martarelli, C. S., \& Mast, F. W. (2013). Eye movements during longterm pictorial recall. Psychological Research, 77(3), 303-309.

Martinez-Conde, S., Macknik, S. L., Troncoso, X. G., \& Dyar, T. A. (2006). Microsaccades counteract visual fading during fixation. Neuron, 49(2), 297-305. doi:10.1016/j.neuron.2005.11.033.

Masson, N., \& Pesenti, M. (2015). Interference of lateralized distractors on arithmetic problem solving: A functional role for attention shifts in mental calculation. Psychological Research (online first version).

McCrink, K., Dehaene, S., \& Dehaene-Lambertz, G. (2007). Moving along the number line: Operational momentum in nonsymbolic arithmetic. Perception and Psychophysics, 69(8), 1324-1333.

Meyerhoff, H. S., Moeller, K., Debus, K., \& Nuerk, H.-C. (2012). Multi-digit number processing beyond the two-digit number range: A combination of sequential and parallel processes. Acta Psychologica (Amst), 140(1), 81-90.

Micic, D., Ehrlichman, H., \& Chen, R. (2010). Why do we move our eyes while trying to remember? The relationship between nonvisual gaze patterns and memory. Brain and Cognition, 74(3), $210-224$

Mock, J., Huber, S., Klein, E., \& Moeller, K. (2016). Insights into numerical cognition: Considering eye-fixations in number processing and arithmetic. Psychological Research, (online first version).

Moeller, K., Fischer, M. H., Nuerk, H. C., \& Willmes, K. (2009). Sequential or parallel decomposed processing of two-digit numbers? Evidence from eye-tracking. Quarterly Journal of Experimental Psychology (Hove), 62(2), 323-334. doi:10.1080/ 17470210801946740.

Moeller, K., Shaki, S., Göbel, S. M., \& Nuerk, H.-C. (2015). Language influences number processing: A quadrilingual study. Cognition, 136, 150-155.

Myachykov, A., Ellis, R., Cangelosi, A., \& Fischer, M. H. (2016). Ocular drift along the mental number line. Psychological Research, (online first version).

Nuerk, H.-C., Weger, U., \& Willmes, K. (2002). A unit-decade compatibility effect in German number words. Current Psychology Letters: Behaviour, Brain \& Cognition, 7, 19-38.

Nyffeler, T., Hartmann, M., Hess, C. W., \& Müri, R. M. (2008). Visual vector inversion during memory antisaccades: A TMS study. Progress in Brain Research, 171, 429-432.
Pinhas, M., \& Fischer, M. H. (2008). Mental movements without magnitude? A study of spatial biases in symbolic arithmetic. Cognition, 109(3), 408-415. doi:10.1016/j.cognition.2008.09. 003.

Ranzini, M., Lisi, M., \& Zorzi, M. (2016). Voluntary eye movements direct attention on the mental number space. Psychological Research, (online first version).

Rau, A. K., Moll, K., Snowling, M. J., \& Landerl, K. (2015). Effects of orthographic consistency on eye movement behavior: German and English children and adults process the same words differently. Journal of Experimental Child Psychology, 130, 92-105.

Rayner, K. (1998). Eye movements in reading and information processing: 20 years of research. Psychological Bulletin, 124(3), 372.

Rayner, K., \& Reingold, E. M. (2015). Evidence for direct cognitive control of fixation durations during reading. Current Opinion in Behavioral Sciences, 1, 107-112.

Reichle, E. D., Pollatsek, A., Fisher, D. L., \& Rayner, K. (1998). Toward a model of eye movement control in reading. Psychological Review, 105(1), 125.

Rugani, R., Vallortigara, G., Priftis, K., \& Regolin, L. (2015). Number-space mapping in the newborn chick resembles humans' mental number line. Science, 347(6221), 534-536.

Schneider, M., Heine, A., Thaler, V., Torbeyns, J., De Smedt, B., Verschaffel, L., \& Stern, E. (2008). A validation of eye movements as a measure of elementary school children's developing number sense. Cognitive Development, 23(3), 409-422.

Schwarz, W., \& Keus, I. M. (2004). Moving the eyes along the mental number line: Comparing SNARC effects with saccadic and manual responses. Perception and Psychophysics, 66(4), $651-664$.

Schwarz, W., \& Müller, D. (2006). Spatial associations in numberrelated tasks: A comparison of manual and pedal responses. Experimental Psychology, 53(1), 4-15.

Shaki, S., \& Fischer, M. H. (2015). Newborn chicks need no number tricks. Commentary: Number-space mapping in the newborn chick resembles humans' mental number line. Frontiers in Human Neuroscience, 9, 451.

Shaki, S., Fischer, M. H., \& Petrusic, W. M. (2009). Reading habits for both words and numbers contribute to the SNARC effect. Psychonomic Bulletin \& Review, 16(2), 328-331. doi:10.3758/ PBR.16.2.328.

Siegenthaler, E., Costela, F. M., McCamy, M. B., Di Stasi, L. L., Otero-Millan, J., Sonderegger, A., \& Martinez-Conde, S. (2014). Task difficulty in mental arithmetic affects microsaccadic rates and magnitudes. European Journal of Neuroscience, 39, 287-294.

Siegler, R. S., \& Opfer, J. E. (2003). The development of numerical estimation evidence for multiple representations of numerical quantity. Psychological Science, 14(3), 237-250.

Spivey, M. J., \& Geng, J. J. (2001). Oculomotor mechanisms activated by imagery and memory: Eye movements to absent objects. Psychological Research, 65(4), 235-241.

Starr, M. S., \& Rayner, K. (2001). Eye movements during reading: Some current controversies. Trends in Cognitive Science, 5(4), 156-163.

Sullivan, J. L., Juhasz, B. J., Slattery, T. J., \& Barth, H. C. (2011). Adults' number-line estimation strategies: Evidence from eye movements. Psychonomic Bulletin \& Review, 18(3), 557-563. doi:10.3758/s13423-011-0081-1.

Susac, A. N. A., Bubic, A., Kaponja, J., Planinic, M., \& Palmovic, M. (2014). Eye movements reveal students' strategies in simple equation solving. International Journal of Science and Mathematics Education, 12(3), 555-577. 
Tanenhaus, M. K., Spivey-Knowlton, M. J., Eberhard, K. M., \& Sedivy, J. C. (1995). Integration of visual and linguistic information in spoken language comprehension. Science, 268(5217), 1632-1634.

Thomas, L. E., \& Lleras, A. (2007). Moving eyes and moving thought: On the spatial compatibility between eye movements and cognition. Psychonomic Bulletin \& Review, 14(4), 663-668.

van Viersen, S., Slot, E. M., Kroesbergen, E. H., Van't Noordende, J. E., \& Leseman, P. P. (2013). The added value of eye-tracking in diagnosing dyscalculia: A case study. Frontiers in Psychology, 4, 679. doi:10.3389/fpsyg.2013.00679.

van't Noordende, J. E., van Hoogmoed, A. H., Schot, W. D., Kroesbergen, E. H. (2016). Number line estimation strategies in children with mathematical learning difficulties measured by eye tracking. Psychological Research, (online first version).

Wade, N., \& Tatler, B. W. (2005). The moving tablet of the eye: The origins of modern eye movement research. New York, NY: Oxford University Press.
Walsh, V. (2003). A theory of magnitude: Common cortical metrics of time, space and quantity. Trends in Cognitive Science, 7(11), 483-488.

Walsh, V. (2015). A theory of magnitude: The parts that sum to number. In R. C. Kadosh \& A. Dowker (Eds.), The Oxford Handbook of Numerical Cognition (pp. 552-565 ). Oxford, U.K.: Oxford University Press.

Winter, B., Matlock, T., Shaki, S., \& Fischer, M. H. (2015). Mental number space in three dimensions. Neuroscience and Biobehavioral Reviews, 57, 209-219.

Wynn, K. (1992). Addition and subtraction by human infants. Nature, 358(6389), 749-750.

Yu, X., Liu, J., Li, D., Liu, H., Cui, J., \& Zhou, X. (2016). Dynamic mental number line in simple arithmetic. Psychological Research, (online first version).

Zorzi, M., Priftis, K., \& Umilta, C. (2002). Brain damage: Neglect disrupts the mental number line. Nature, 417(6885), 138-139. doi:10.1038/417138a. 